\title{
Where's the leadership? Future commitments of Unicef and WHO for global child health
}

\author{
(c) $(\mathcal{O} \Theta$ OPEN ACCESS
}

The authors identified some errors in this article (BMJ 2018;362:k3219, doi:10.1136/bmj.k3219). In the section on potential solutions and barriers to success, the location of the October 2018 review meeting will be Astana not Almaty. In the section on the WHO-Unicef leadership strategy, the members of the H6 group were incorrect and should include the World Bank and not the UN Foundation. In addition GFF stands for Global Financing Facility not Global Finance Facility. The article has been updated. 